\title{
RATIONAL PERIOD FUNCTIONS OF THE MODULAR GROUP II
}

\author{
by MARVIN I. KNOPP $\dagger$
}

(Received 30 January 1980)

1. Introduction. In the earlier article [7], I began the study of rational period functions for the modular group $\Gamma(1)=S L(2, \mathbf{Z})$ (regarded as a group of linear fractional transformations) acting on the Riemann sphere. These are rational functions $q(z)$ which occur in functional equations of the form

$$
F(z+1)=F(z), \quad z^{-2 k} F(-1 / z)=F(z)+q(z),
$$

where $k \in \mathbf{Z}$ and $F$ is a function meromorphic in the upper half-plane $\mathscr{H}$, restricted in growth at the parabolic cusp $\infty$. The growth restriction may be phrased in terms of the Fourier expansion of $F(z)$ at $\infty$ :

$$
F(z)=\sum_{n=\mu}^{\infty} a_{n} e^{2 \pi i n z}, \quad y=\operatorname{Im} z>y_{0} \geq 0,
$$

with some $\mu \in \mathbf{Z}$. If (1.1) and (1.2) hold, then we call $F$ a modular integral of weight $2 k$ and $q(z)$ the period of $F$.

Frequently-in particular when $k>0$-it entails no loss of generality to assume that $F$ is holomorphic in $\mathscr{H}$ and $\mu \geq 0$. In this instance, (1.2) holds in all of $\mathscr{H}\left(y_{0}=0\right)$ and $F$ satisfies the growth condition (see [8, pp. 622-623])

$$
|F(z)| \leq K\left(|z|^{\alpha}+y^{-\beta}\right), \quad z \in \mathscr{H},
$$

for some $K, \alpha, \beta>0$. If $F$ is a modular integral of weight $2 k$ and if in addition it is holomorphic in $\mathscr{H}$ and $\mu \geq 0$ in (1.2)-that is to say, (1.3) holds-it is called an entire modular integral of weight $2 k$. It is a simple matter to verify that for $F$ defined by (1.2) with $\mu \geq 0,(1.3)$ is equivalent to

$$
a_{n}=O\left(n^{\gamma}\right), \quad n \rightarrow+\infty,
$$

for some $\gamma>0$. Thus an entire modular integral is a modular integral for which $\mu \geq 0$ in (1.2) and (1.4) holds.

When $k \leq 0$ a familiar case of (1.1) occurs with $q(z)$ a polynomial of degree $\leq-2 k$, the period polynomial which arises in the Eichler cohomology theory $[3,4,6,8]$. In addition to these $q(z)=c / z$ arises naturally as the period function of the logarithmic derivative of a modular form of any nonzero weight. It occurs as well in the functional equation of $E_{2}(z)$, the Eisenstein series of weight 2 on $\Gamma(1)$. (See for example [5].)

In [7, Theorem 1] I introduced the new rational period functions for $\Gamma(1)$

$$
q_{2 k}(z)=\left(z-z_{0}\right)^{-k}\left(z+z_{0}^{\prime}\right)^{-k}+\left(z+z_{0}\right)^{-k}\left(z-z_{0}^{\prime}\right)^{-k},
$$

†This research was supported in part by National Science Foundation grant MCS-7903471.

Glasgow Math. J. 22 (1981) 185-197. 
of weight $2 k$ (called weight $-2 k$ in [7]), where $k$ is odd and $z_{0}=(\sqrt{5}+1) / 2, z_{0}^{\prime}=z_{0}^{-1}=$ $(\sqrt{5}-1) / 2$; when $k \leq 0, q_{2 k}(z)$ is a polynomial of degree $-2 k$. Applying Hecke operators, we showed [7, Theorem 3] that with $k>0$ and $k$ odd, there exists an infinite set of linearly independent rational period functions of weight $2 k$ on $\Gamma(1)$, all of them with poles of order $k$ lying in $\mathbf{Q}(\sqrt{5})$. Very recently, Alayne Parson has applied results on the Hecke groups to find new rational period functions for $\Gamma(1)$, with poles lying in $\mathbf{Q}(\sqrt{3})$ and in $\mathbf{Q}(\sqrt{21})$, again if $k>0$ and $k$ is odd [9].

On the other hand, I show here (Theorem 1(a), below) that a pole of any rational period function $q(z)$ must lie in $\mathbf{Q}^{+}(\sqrt{n}), n \in \mathbf{Z}^{+}$, where $\mathrm{Q}^{+}=\mathbf{Q} \cup \infty$; indeed every such $q(z)$ can be decomposed into a sum of rational period functions, each with its poles in a fixed quadratic extension of $\mathbf{Q}$. That is to say,

$$
q(z)=q^{(1)}(z)+q^{(2)}(z)+q^{(3)}(z)+q^{(5)}(z)+\ldots,
$$

where $q^{(m)}(z)$ is a rational period function, $q^{(1)}(z)$ has its poles in $\mathbf{Q}^{+}$and $q^{(m)}(z), m$ squarefree and $\geq 2$, has its poles in $\mathbf{Q}(\sqrt{m})-\mathbf{Q}$. I determine in addition the explicit form of $q^{(1)}(z)$ (poles in $\mathbf{Q}^{+}$), showing in particular that the only possible rational poles are $0(k>0)$ or 0 and $\infty(k \leq 0)$. This is the content of Theorem 1(b), and Theorem 2. It remains an open question whether there are rational period functions with poles in $\mathbf{Q}(\sqrt{n})$, $n \geq 2$, when $k \geq 0$ is even and when $k<0$.

We close the paper with some observations regarding Mellin transforms of entire modular integrals and, in particular, the functional equations that they satisfy. It turns out that if the poles of $q(z)$ lie in $\mathbf{Q}^{+}$(i.e. at 0 or $\infty$ ), then the Mellin transform of the corresponding entire modular integral satisfies the same type of functional equation as does the Mellin transform of an entire modular form (Theorem 3). There is a converse as well, showing that when the Mellin transform of an entire modular integral possesses such a functional equation, then the poles of the associated period function $q(z)$ must lie in $\mathbf{Q}^{+}$. This converse explains the fact that the Mellin transform studied in $[7, \S 3]$ does not satisfy the usual type of functional equation (see [7, Theorem 2]), since it arises from a modular integral whose period function has poles in $\mathbf{Q}(\sqrt{5})-\mathbf{Q}$.

2. The Poles. $\Gamma(1)$ is generated by the matrices $S=\left(\begin{array}{ll}1 & 1 \\ 0 & 1\end{array}\right), T=\left(\begin{array}{cc}0 & -1 \\ 1 & 0\end{array}\right)$, with the relations $T^{2}=(S T)^{3}=I$. This fact and (1.1) together imply that

$$
\begin{aligned}
& \text { (a) } z^{-2 k} q(-1 / z)+q(z)=0 \\
& \text { (b) }(z-1)^{-2 k} q\left(-\frac{1}{z-1}\right)+z^{-2 k} q\left(\frac{z-1}{z}\right)+q(z)=0 .
\end{aligned}
$$

These two identities yield the further one

$$
(z+1)^{-2 k} q\left(\frac{z}{z+1}\right)+q(z+1)=q(z) .
$$

As we observe in [7, p. 49], the identities in (2.1) are, conversely, sufficient for the 
existence of a function $F$ holomorphic in $\mathscr{\ell}$ and satisfying (1.1) and (1.2). Furthermore, when $k>0$ we can construct $F$ with $\mu \geq 0$ in (1.2).

We shall apply (2.1) and (2.2) to find necessary conditions on the poles of $q(z)$.

THEOREM 1. (a) If $z_{0}$ is a finite pole of any rational function satisfying (2.1) then there is a squarefree positive integer $n$ such that $z_{0} \in \mathbf{Q}(\sqrt{n})$.

(b) If the finite pole $z_{0}$ of $q(z)$ is in $\mathbf{Q}$, then $z_{0}=0$.

REMARK. In particular $z_{0}$ is real, a conclusion valid even if the function $F(z)$ of (1.1) has poles in $\mathscr{H}$.

Proof. (a). We are assuming $z_{0} \neq \infty$. Clearly, we may assume as well that $z_{0} \notin \mathbf{Q}$, so that in particular $z_{0} \neq 0,-1, \infty$. By (2.2), either $z_{0}+1$ or $z_{0} /\left(z_{0}+1\right)$ is a pole of $q(z)$. Thus when $z_{0}$ is a pole, so is $M_{1} z_{0}$, where $M_{1}$ is either

$$
\left(\begin{array}{ll}
1 & 1 \\
0 & 1
\end{array}\right) \text { or }\left(\begin{array}{ll}
1 & 0 \\
1 & 1
\end{array}\right)
$$

Applying (2.2) once more we find that $M_{2} M_{1} z_{0}$ is a pole, where again $M_{2}$ is either

$$
\left(\begin{array}{ll}
1 & 1 \\
0 & 1
\end{array}\right) \text { or }\left(\begin{array}{ll}
1 & 0 \\
1 & 1
\end{array}\right) \text {. }
$$

We continue in this fashion to find after $k$ steps that $M_{k} M_{k-1} \ldots M_{1} z_{0}$ is a pole of $q(z)$, where each $M_{j}$ is either

$$
\left(\begin{array}{ll}
1 & 1 \\
0 & 1
\end{array}\right) \text { or }\left(\begin{array}{ll}
1 & 0 \\
1 & 1
\end{array}\right)
$$

But since both matrices have trace 2 and nonnegative entries, any product of them has nonnegative entries and trace $\geq 2$, that is, such a product represents either a parabolic or a hyperbolic transformation. (To illustrate the point, observe that for $k=2$ the matrices that arise are

$$
\left.\left(\begin{array}{ll}
1 & 3 \\
0 & 1
\end{array}\right),\left(\begin{array}{ll}
2 & 3 \\
1 & 2
\end{array}\right),\left(\begin{array}{ll}
3 & 2 \\
1 & 1
\end{array}\right),\left(\begin{array}{ll}
3 & 1 \\
2 & 1
\end{array}\right),\left(\begin{array}{ll}
1 & 2 \\
1 & 3
\end{array}\right),\left(\begin{array}{ll}
1 & 1 \\
2 & 3
\end{array}\right),\left(\begin{array}{ll}
2 & 1 \\
3 & 2
\end{array}\right),\left(\begin{array}{ll}
1 & 0 \\
3 & 1
\end{array}\right)\right)
$$

Since $q$ is rational it has only finitely many poles; hence there exist $k, \ell \in \mathbf{Z}, k>\ell \geq 0$, with $M_{k} M_{k-1} \ldots M_{1} z_{0}=M_{\ell} M_{\ell-1} \ldots M_{1} z_{0}$, which is to say that $z_{0}^{\prime}=M_{\ell} \ldots M_{1} z_{0}$ is a fixed point of $M_{k} \ldots M_{\ell+1}$. Putting $M_{k} \ldots M_{\ell+1}=\left(\begin{array}{cc}a & b \\ c & d\end{array}\right)$, we have $a, d>0, b, c \geq 0$ and $a d-b c=$ 1. Then, $z_{0}^{\prime}=\left(a z_{0}^{\prime}+b\right) /\left(c z_{0}^{\prime}+d\right)$, or

$$
z_{0}^{\prime}=\frac{a-d \pm \sqrt{ }\left\{(d+a)^{2}-4\right\}}{2 c} .
$$

But $(d+a)^{2}-4 \geq 0$, so either $z_{0}^{\prime} \in \mathbf{Q}$ or $z_{0}^{\prime} \in \mathbf{Q}(\sqrt{n})$ for some squarefree $n \in \mathbf{Z}^{+}$. But $z_{0}^{\prime} \in \mathbf{Q}$ implies $z_{0} \in \mathbf{Q}$, a contradiction to our earlier assumption. Thus $z_{0}^{\prime} \in \mathbf{Q}(\sqrt{n})$ and consequently $z_{0}=\left(M_{\ell} \ldots M_{1}\right)^{-1} z_{0}^{\prime} \in \mathbf{Q}(\sqrt{n})$. 
(b). Now assume $z_{0} \in \mathbf{Q}$ and $z_{0} \neq 0$. If $z_{0}<0$ then by $(2.1 a)-1 / z_{0}>0$ is again a rational pole of $q(z)$. Thus we may assume from the outset that $z_{0}>0$ and under this assumption the procedure of $(a)$ shows once again that $z_{0}^{\prime}=M_{\ell} \ldots M_{1} z_{0}\left(\ell \in \mathbf{Z}^{+}\right)$has the form

$$
z_{0}^{\prime}=\frac{a-d \pm \sqrt{ }\left\{(d+a)^{2}-4\right\}}{2 c}, \quad a, d \in \mathbf{Z}^{+} .
$$

But $z_{0} \in \mathbf{Q}$ implies $z_{0}^{\prime} \in \mathbf{Q}$, so that $(d+a)^{2}-4$ is a square and thus $a=d=1$. It follows that $z_{0}^{\prime}=0$, a contradiction to $z_{0}^{\prime}=M_{\ell} \ldots M_{1} z_{0}>0$. The proof is complete.

We strengthen Theorem 1(b) with

THEOREM 2. If the poles of $q(z)$ lie in $\mathbf{Q}^{+}$then

$$
q(z)=\left\{\begin{array}{l}
b_{0}\left(1-z^{-2 k}\right) \quad \text { if } \quad k>1 \\
b_{0}\left(1-z^{-2}\right)+b_{1} z^{-1} \quad \text { if } \quad k=1 \\
b_{0}\left(z^{-1}+z^{-2 k+1}\right)+p_{k}(z) \text { if } k \leq 0
\end{array}\right.
$$

where $b_{0}, b_{1}$ are complex numbers and $p_{k}(z)$ is a polynomial in $z$ of degree at most $-2 k$.

Proof. (i). The case $k>0$. By Theorem 1(b) we may write

$$
q(z)=a_{\ell} z^{-\ell}+\ldots+a_{1} z^{-1}+b_{0}+b_{1} z+\ldots+b_{m} z^{m},
$$

with $\ell, m \geq 0$. Applying (2.1a) and comparing like terms, we find that $\ell=m+2 k$. We insert the expression (2.4) into (2.2) and compare the principal part at $\infty$ on the two sides. This yields

$$
b_{0}+b_{1}(z+1)+\ldots+b_{m}(z+1)^{m}=b_{0}+b_{1} z+\ldots+b_{m} z^{m},
$$

so that $b_{1}=b_{2}=\ldots=b_{m}=0, \ell=2 k$ and

By $(2.1 a)$ we find

$$
q(z)=a_{2 k} z^{-2 k}+\ldots+a_{1} z^{-1}+b_{0} .
$$

$$
a_{2 k}-a_{2 k-1} z^{-1}+\ldots+(-1)^{j} a_{2 k-j} z^{-j}+\ldots+b_{0} z^{-2 k}=-a_{2 k} z^{-2 k}-a_{2 k-1} z^{-2 k+1}-\ldots-b_{0},
$$

so that

$$
b_{0}=-a_{2 k}, a_{2 k-j}=(-1)^{j+1} a_{j} \text { for } 1 \leq j \leq k .
$$

In particular, $a_{k}=(-1)^{k+1} a_{k}$, so $a_{k}=0$ if $k$ is even.

Application of $(2.1 b)$ to $(2.5)$ leads to

$$
\begin{aligned}
a_{2 k}-a_{2 k-1}(z-1)^{-1}+\ldots+(-1)^{j} a_{2 k-j}(z-1)^{-j} & +\ldots+b_{0}(z-1)^{-2 k}+ \\
+a_{2 k}(z-1)^{-2 k}+a_{2 k-1} z^{-1}(z-1)^{1-2 k}+\ldots+ & +a_{j} z^{-j}(z-1)^{j-2 k}+\ldots+b_{0} z^{-2 k} \\
& +a_{2 k} z^{-2 k}+\ldots+a_{1} z^{-1}+b_{0}=0 .
\end{aligned}
$$

In (2.7) the coefficient of $z^{-2 k+1}$ is $a_{2 k-1}-a_{1}$, so that $a_{2 k-1}=a_{1}$, a fact already contained 
in (2.6). Considering the coefficient of $z^{-2 k+2}$, we find that $a_{2 k-2}-a_{1}+a_{2}=0$. But by (2.6) $a_{2 k-2}+a_{2}=0$, so that $a_{1}=a_{2 k-1}=0$. The coefficient of $z^{-2 k+3}$ in (2.7) is $a_{2 k-3}-a_{1}+2 a_{2}$ $a_{3}=a_{2 k-3}+2 a_{2}-a_{3}=0$. But (2.6) implies $a_{2 k-3}=a_{3}$, from which follow $a_{2}=0$ and $a_{2 k-2}=0$. Continuing in this manner we conclude that $a_{j}=a_{2 k-j}=0$ for $1 \leq j<k$. Thus $q(z)$ has the form

$$
q(z)=\left\{\begin{array}{l}
b_{0}\left(1-z^{-2 k}\right), \quad \text { if } k \text { is even } \\
b_{0}\left(1-z^{-2 k}\right)+a_{k} z^{-k}, \quad \text { if } k \text { is odd. }
\end{array}\right.
$$

Suppose $k$ is odd. It remains to show that in the second form, $a_{k} \neq 0$ is possible only for $k=1$. Since $b_{0}\left(1-z^{-2 k}\right)$ satisfies (2.1) it is sufficient to prove that $z^{-k}$ satisfies (2.1) if and only if $k=1$. But $z^{-k}$ satisfies $(2.1 a)$ for any $k \in Z$, so we consider $(2.1 b)$. With $q(z)=z^{-k}$, we have

$$
\begin{aligned}
(z-1)^{-2 k} q\left(-\frac{1}{z-1}\right)+z^{-2 k} q\left(\frac{z-1}{z}\right)+q(z) & =-(z-1)^{-k}+z^{-k}(z-1)^{-k}+z^{-k} \\
& =z^{-k}\left\{1+\frac{1-z^{k}}{(z-1)^{k}}\right\},
\end{aligned}
$$

and this is 0 only when $k=1$.

(ii) The case $k \leq 0$. In this instance we apply the observation of $\mathrm{G}$. Bol [2] that

$$
D^{(r+1)}\left\{(c z+d)^{r} f\left(\frac{a z+b}{c z+d}\right)\right\}=(c z+d)^{-r-2} f^{(r+1)}\left(\frac{a z+b}{c z+d}\right)
$$

for $r \in \mathbf{Z}, r \geq 0$ and $a d-b c=1$, whenever the derivatives in question exist. Let $q(z)$ be a rational period function of weight $2 k, k \leq 0$. Differentiating the relations in $(2.1)-2 k+1$ times and applying (2.8) with $r=-2 k$, we find that $\left.q^{(-2 k+1}\right)(z)$ is a rational period function of weight $-2 k+2>0$. By part (i) of the proof, $q^{(-2 k+1)}(z)=b_{0}\left(1-z^{2 k-2}\right)$, since the term $b_{1} z^{-1}$ does not occur as the derivative of a rational function. Integrating $-2 k+1$ times, we conclude that $q(z)$ has the form $b_{0}^{\prime}\left(z^{-2 k+1}+z^{-1}\right)+p_{k}(z)$, with $p_{k}(z)$ a polynomial of degree $\leq-2 k$. The proof is complete.

REMARKS. (i) The period functions $q$ described in Theorem 3 actually occur. When $k=0$, however, such a $q(z) \equiv 0$, a fact which becomes evident upon application of (2.1). For any $k \in \mathbf{Z}, b_{0}\left(1-z^{-2 k}\right)$ may be regarded as the "trivial" rational period function, since it arises with $F(z)=b_{0}$ in (1.1).

(ii) If $E_{2 k}(z)$ is the Eisenstein series of weight $2 k$ on (1), then $E_{2}(z)$ has the rational period function $b_{1} z^{-1}$, as remarked earlier, while for $k>1 E_{2 k}(z)$ is a genuine modular form (that is, the period function is 0$)$. If, however, $I_{2 k}(z)$ is a $(2 k-1)$-fold integral of $E_{2 k}(z)$, so normalized that

$$
(2 k-1) ! I_{2 \mathrm{k}}(z)=z^{2 k-1}+\sum_{n=1}^{\infty} c_{n} e^{2 \pi i n z},
$$

then $H_{2 k}(z)=(2 k-1) ! I_{2 k}(z)-z^{2 k-1}$ is a modular integral (in the sense of $(1.1)$ ) of weight 
$-2 k+2$ with period function of the form

$$
z^{-1}+z^{2 k-1}+\psi_{k}(z)
$$

with $\psi_{k}(z)$ a polynomial of degree $\leq 2 k-2$. With $k$ fixed, the difference of two period functions of the form (2.9) is a period polynomial (of degree $\leq 2 k-2$ ) of the type first studied by Eichler in his classic paper [4]. One can show from (2.1) in an elementary way that for $1 \leq k \leq 5$ such a polynomial must have the trivial form $b_{0}\left(z^{2 k-2}-1\right)$.

(iii) In recent correspondence Dr. Parson reports that for low negative weights she has determined the general rational period functions for $\Gamma(1)$, with poles in $\mathbf{Q}^{+}$. They are (cf. Theorem 2):

$$
\begin{aligned}
h_{4}(z) & =z^{-1}-5 z+z^{3}+c\left(z^{2}-1\right), \quad \text { weight }-2 ; \\
h_{6}(z) & =z^{-1}-\frac{7}{2} z-\frac{7}{2} z^{3}+z^{5}+c\left(z^{4}-1\right), \quad \text { weight }-4 ; \\
h_{8}(z) & =z^{-1}-\frac{10}{3}\left(z+z^{5}\right)-\frac{7}{3} z^{3}+z^{7}+c\left(z^{6}-1\right), \quad \text { weight }-6 ; \\
h_{10}(z) & =z^{-1}-\frac{33}{10}\left(z+z^{7}\right)-\frac{11}{5}\left(z^{3}+z^{5}\right)+z^{9}+c\left(z^{8}-1\right), \quad \text { weight }-8 ; \\
h_{12}(z) & =z^{-1}-\frac{91}{4}\left(z^{3}+z^{7}\right)+\frac{65}{2} z^{5}+z^{11}+c_{0}\left(z^{10}-1\right)+c_{2}\left(z^{8}-3 z^{6}+3 z^{4}-z^{2}\right), \quad \text { weight }-10 .
\end{aligned}
$$

By the observation made in Remark (ii) above, $h_{2 k}(z)$ is the period function of $H_{2 k}(z)+$ constant, so long as $1 \leq k \leq 5$. The situation is more complex for $h_{12}(z)$, however. In [7] we showed that

$$
q_{-10}(z)=2\left(z^{10}+5 z^{8}-15 z^{6}+15 z^{4}-5 z^{2}-1\right)
$$

is an Eichler period polynomial of weight -10 for $\Gamma(1)$. (By the principal result of [3], $q_{-10}(z)$ cannot be the period polynomial of a cusp form.) From the expression for $h_{12}(z)$ and the form of $q_{-10}(z)$ it follows that

$$
z^{-1}-\frac{91}{4}\left(z^{3}+z^{7}\right)+\frac{65}{2} z^{5}+z^{11}
$$

is again a rational period function of weight -10 for $\Gamma(1)$, very likely the one corresponding to $H_{12}(z)$. Note that $q_{-10}(z)$ is obtained from the last two terms of $h_{12}(z)$, with $c_{0}=2$ and $c_{2}=5$.

3. Mellin transforms. In this section we deal with entire modular integrals only, as these are the ones to which it is possible to associate a Dirichlet series by means of the Mellin transform. Thus we assume from now on that $\left\{a_{n} \mid n=0,1,2,3, \ldots,\right\}$ is a sequence of complex numbers satisfying (1.4), that is, $a_{n}=0\left(n^{\gamma}\right), n \rightarrow+\infty$, for some $\gamma>0$. We shall consider the power series $F(z)=\sum_{n=0}^{\infty} a_{n} e^{2 \pi i n z}$ and the Dirichlet series

$$
\Phi(s)=\Phi_{F}(s)=(2 \pi)^{-s} \Gamma(s) \sum_{n=1}^{\infty} a_{n} n^{-s},
$$


related to it by the Mellin transform:

$$
\Phi_{F}(s)=\int_{0}^{\infty}\left\{F(i y)-a_{0}\right\} y^{s-1} d y .
$$

(Here $\Gamma(s)$ is the gamma-function.) Since (1.3) and (1.4) hold it is possible to carry out termwise integration in (3.2) to obtain the series (3.1), which of necessity converges in some half-plane.

THEOREM 3. Suppose $F(z)$ is an entire modular integral of weight $2 k$ such that its associated rational period function has poles only in $\mathbf{Q}^{+}$(thus at 0 and $\infty$, by Theorem $1(b)$.). Then

(a) $\Phi(s)=\Phi_{F}(s)$ has an analytic continuation to the entire s-plane, except for possible simple poles at

$$
\begin{aligned}
& s=0 \text { and } s=2 k, \text { when } k>1 \\
& s=0,1 \text { and } 2, \text { when } k=1 \\
& s=2 k-1,2 k, \ldots 0,1, \text { when } k \leq 0
\end{aligned}
$$

(b) In every case

$$
\Phi(2 k-s)=(-1)^{k} \Phi(s),
$$

and $\Phi(s)$ is bounded in each "truncated strip" of the form $\sigma_{1} \leq \operatorname{Re} s \leq \sigma_{2},|\operatorname{Im} t| \geq t_{0}>0$, uniformly in $\sigma_{1}$ and $\sigma_{2}$.

REMARK. Here, the case $k=1$ and $k \leq 0$ come into consideration, whereas nontrivial entire modular forms of weight $2 k$ exist only when $k>1$. Note that when $k \leq 0$, the poles of $\Phi_{F}(s)$ are not quite confined to the "critical strip" $2 k \leq \sigma \leq 0$, but may occur at $s=2 k-1$ and $s=1$ as well.

Proof. Suppose $F(z)$ is an entire modular integral of weight $2 k$ with rational period function $q(z)$ and the poles of $q(z)$ lie in $\mathbf{Q}^{+}$. Thus we have

$$
\begin{aligned}
& \text { (i) } F(z)=\sum_{n=0}^{\infty} a_{n} e^{2 \pi i n z}, \quad z \in \mathscr{H}, \\
& \text { (ii) } a_{n}=O\left(n^{\gamma}\right), \gamma>0, \text { as } n \rightarrow+\infty, \\
& \text { (iii) } z^{-2 k} F(-1 / z)=F(z)+q(z) .
\end{aligned}
$$

Let $\Phi_{F}(s)=\Phi(s)$ be defined by (3.2), the integral converging for $\sigma=\operatorname{Re} s$ sufficiently large by virtue of (1.3) and (3.4i).

For large $\sigma$, we may then write

$$
\Phi(s)=\int_{1}^{\infty}\left\{F(i y)-a_{0}\right\} y^{s-1} d y+\int_{0}^{1}\left\{F(i y)-a_{0}\right\} y^{s-1} d y .
$$


Rewrite the second integral by making use of (1.1):

$$
\begin{aligned}
\int_{0}^{1}\left\{F(i y)-a_{0}\right\} y^{s-1} d y= & \int_{1}^{\infty}\left\{F(i / y)-a_{0}\right\} y^{-s-1} d y \\
= & (-1)^{k} \int_{1}^{\infty}\{F(i y)+q(i y)\} y^{-s-y+2 k} d y-\frac{a_{0}}{s} \\
= & (-1)^{k} \int_{1}^{\infty}\left\{F(i y)-a_{0}\right\} y^{-s-1+2 k} d y+(-1)^{k} a_{0} \int_{1}^{\infty} y^{-s-1+2 k} d y \\
& -\frac{a_{0}}{s}+(-1)^{k} \int_{1}^{\infty} q(i y) y^{-s-1+2 k} d y \\
= & (-1)^{k} \int_{1}^{\infty}\left\{F(i y)-a_{0}\right\} y^{-s-1+2 k} d y+\frac{(-1)^{k} a_{0}}{s-2 k} \\
& -\frac{a_{0}}{s}+(-1)^{k} \int_{1}^{\infty} q(i y) y^{-s-1+2 k} d y .
\end{aligned}
$$

Hence,

$$
\Phi(s)=D_{1}(s)+(-1)^{k} D_{2}(s)+a_{0}\left(\frac{(-1)^{k}}{s-2 k}-\frac{1}{s}\right)+(-1)^{k} E(s),
$$

where

$$
\begin{gathered}
D_{1}(s)=\int_{1}^{\infty}\left\{F(i y)-a_{0}\right\} y^{s-1} d y, \quad D_{2}(s)=\int_{1}^{\infty}\left\{F(i y)-a_{0}\right\} y^{-s-1+2 k} d y, \quad \text { and } \\
E(s)=\int_{1}^{\infty} q(i y) y^{-s-1+2 k} d y .
\end{gathered}
$$

From (3.4i) it follows that $D_{1}(s)$ and $D_{2}(s)$ are entire functions of $s$ and from their form it is evident that

$$
D_{1}(2 k-s)=D_{2}(s), \quad D_{2}(2 k-s)=D_{1}(s) .
$$

By Theorem 1(b), $q(z)$ has the form $q(z)=\sum_{n=-K}^{N} \alpha_{n} z^{n}$, and it follows that

$$
E(s)=\sum_{n=-K}^{N} \alpha_{n}(i)^{n} \frac{1}{s-2 k-n},
$$

so long as $\operatorname{Re} s>2 k+N$. For more explicit information about $E(s)$ we consider the three cases $k>1, k=1, k \leq 0$.

(i) Let $k>1$. By Theorem 2, $q(z)=b_{0}\left(1-z^{-2 k}\right)$, so that

$$
E(s)=b_{0}\left\{\frac{1}{s-2 k}-\frac{(-1)^{k}}{s}\right\} \text {. }
$$


Thus,

$$
\Phi(s)=D_{1}(s)+(-1)^{k} D_{2}(s)+\left(a_{0}+b_{0}\right)\left\{\frac{(-1)^{k}}{s-2 k}-\frac{1}{s}\right\}
$$

and $\Phi(s)$ has been extended analytically to the entire $s$-plane, with possible simple poles at $s=0, s=2 k$. Furthermore,

$$
\begin{aligned}
\Phi(2 k-s) & =D_{1}(2 k-s)+(-1)^{k} D_{2}(2 k-s)+\left(a_{0}+b_{0}\right)\left\{\frac{(-1)^{k}}{-s}-\frac{1}{2 k-s}\right\} \\
& =D_{2}(s)+(-1)^{k} D_{1}(s)+\left(a_{0}+b_{0}\right)\left\{\frac{1}{s-2 k}-\frac{(-1)^{k}}{s}\right\} \\
& =(-1)^{k} \Phi(s),
\end{aligned}
$$

by (3.6) and (3.7).

(ii) Suppose $k=1$. By Theorem 2, $q(z)=b_{0}\left(1-z^{-2}\right)+b_{1} z^{-1}$, so that

$$
E(s)=\int_{1}^{\infty} q(i y) y^{-s-1+2 k} d y=b_{0}\left(\frac{1}{s-2}+\frac{1}{s}\right)-b_{1} \frac{i}{s-1} .
$$

Then we have

$$
\Phi(s)=D_{1}(s)-D_{2}(s)-a_{0}\left(\frac{1}{s-2}+\frac{1}{s}\right)-b_{0}\left(\frac{1}{s-2}+\frac{1}{s}\right)+b_{1} \frac{i}{s-1},
$$

and the right hand side is analytic in the $s$-plane, with $s=0,1$ and 2 deleted. Consequently,

$$
\begin{aligned}
\Phi(2-s) & =D_{1}(2-s)-D_{2}(2-s)-\left(a_{0}+b_{0}\right)\left(-\frac{1}{s}+\frac{1}{2-s}\right)+b_{1} \frac{i}{1-s} \\
& =D_{2}(s)-D_{1}(s)+\left(a_{0}+b_{0}\right)\left(\frac{1}{s}+\frac{1}{s-2}\right)-b_{1} \frac{i}{s-1} \\
& =-\Phi(s),
\end{aligned}
$$

by (3.6).

(iii) Let $k \leq 0$. Theorem 2 implies that $q(z)=b_{0}\left(z^{-1}+z^{-2 k+1}\right)+\sum_{n=0}^{-2 k} c_{n} z^{n}$, with complex $c_{n}$. Thus,

$$
\begin{aligned}
E(s) & =\int_{1}^{\infty} q(i y) y^{-s-1+2 k} d y \\
& =-i b_{0}\left(\frac{1}{s-2 k+1}+\frac{(-1)^{k+1}}{s-1}\right)+\sum_{n=0}^{-2 k} c_{n}(i)^{n} \frac{1}{s-2 k-n},
\end{aligned}
$$

while the functional equation $z^{-2 k} q(-1 / z)+q(z)=0$ implies further that

$$
c_{-n-2 k}=(-1)^{n+1} c_{n}, \quad 0 \leq n \leq-2 k,
$$


as in the proof of (2.6). We have in this case

$$
\begin{aligned}
\Phi(s)= & D_{1}(s)+(-1)^{k} D_{2}(s)+a_{0}\left\{\frac{(-1)^{k}}{s-2 k}-\frac{1}{s}\right\} \\
& -i b_{0}\left\{\frac{(-1)^{k}}{s-2 k+1}-\frac{1}{s-1}\right\}+(-1)^{k} \sum_{n=0}^{-2 k} c_{n}(i)^{n} \frac{1}{s-2 k-n},
\end{aligned}
$$

a function meromorphic in the entire $s$-plane, with the obvious simple poles. By (3.10) and (3.6),

$$
\begin{aligned}
\Phi(2 k-s)= & D_{2}(s)+(-1)^{k} D_{1}(s)+a_{0}\left\{\frac{1}{s-2 k}-\frac{(-1)^{k}}{s}\right\} \\
& -i b_{0}\left\{\frac{1}{s-2 k+1}-\frac{(-1)^{k}}{s-1}\right\}+(-1)^{k+1} \sum_{n=0}^{-2 k} c_{n}(i)^{n} \frac{1}{s+n} .
\end{aligned}
$$

But,

$$
\begin{aligned}
(-1)^{k+1} \sum_{n=0}^{-2 k} c_{n}(i)^{n} \frac{1}{s+n} & =(-1)^{k+1} \sum_{n=0}^{-2 k} c_{-n-2 k}(i)^{-n-2 k} \frac{1}{s-2 k-n} \\
& =-\sum_{n=0}^{-2 k}(-1)^{n+1} c_{n}(i)^{-n} \frac{1}{s-2 k-n} \\
& =\sum_{n=0}^{-2 k} c_{n}(i)^{n} \frac{1}{s-2 k-n},
\end{aligned}
$$

by (3.9). If we use this expression in (3.11) and compare the result with (3.10) we find that

$$
\Phi(2 k-s)=(-1)^{k} \Phi(s)
$$

as was to be proved.

The boundedness statement follows easily from the representation

$$
\begin{aligned}
\Phi(s)= & \int_{1}^{\infty}\left\{F(i y)-a_{0}\right\} y^{s-1} d y+(-1)^{k} \int_{1}^{\infty}\left\{F(i y)-a_{0}\right\} y^{-s-1+2 k} d y \\
& +a_{0}\left(\frac{(-1)^{k}}{s-2 k}-\frac{1}{s}\right)+(-1)^{k} E(s),
\end{aligned}
$$

if we also take into account the expression

$$
E(s)=\sum_{n=-K}^{N} c_{n}(i)^{n}(s-2 k-n)^{-1} .
$$

This completes the proof.

A converse of Theorem 3 is

THEOREM 4. Suppose $\left\{a_{n}\right\}$ is a sequence of complex numbers satisfying (1.4), so that the Dirichlet series $\sum_{n=1}^{\infty} a_{n} n^{-s}$ converges for $\sigma=\operatorname{Re} s$ sufficiently large. Suppose also that 
$\Phi(s)=(2 \pi)^{-s} \Gamma(s) \sum_{n=1}^{\infty} a_{n} n^{-s}$ can be extended to a function meromorphic in the entire $s$-plane, holomorphic except possibly for simple poles at the rational integers and bounded in every truncated strip of the form $\sigma_{1} \leq \operatorname{Re} s \leq \sigma_{2}$, $|\operatorname{Im} s| \geq t_{0}>0$. If in addition $\Phi(s)$ satisfies the functional equation (3.3) for some integer $k$, then the power series

$$
F(z)=F_{\Phi}(z)=a_{0}+\sum_{n=1}^{\infty} a_{n} e^{2 \pi i n z}
$$

( $a_{0}$ an arbitrary complex number) is an entire modular integral of weight $2 k$, with rational period function having poles only in $\mathbf{Q}^{+}$.

Remarks. (i) By Theorem $1(b)$ the poles of the rational period function $q(z)$ of $F_{\Phi}(z)$ are restricted to 0 and $\infty$.

(ii) Since $\sum_{n=1}^{\infty} a_{n} n^{-s}$ converges in some right half-plane and $\Phi(2 k-s)=(-1)^{k} \Phi(s)$, $\Phi(s)$ has at worst finitely many simple poles at rational integer points. Furthermore, because $F_{\Phi}(z)$ is a modular integral, its period function has the form (2.3). Theorem 3 then shows that this in turn restricts the poles of $\Phi(s)$ to those described in Theorem $3(a)$. This reasoning yields

COROLlaRY 5. Under the restrictions imposed upon $\Phi(s)$ in Theorem $4, \Phi(s)$ has at worst simple poles at $s=0,2 k$, when $k>1 ; s=0,1,2$, when $k=1 ; s=2 k-1,2 k, \ldots, 0,1$, when $k \leq 0$.

(The functional equation $\Phi(2 k-s)=(-1)^{k} \Phi(s)$, which is one of the restrictions imposed upon $\Phi(s)$ here, entails certain obvious relationships among the residues at the poles of $\Phi(s)$. These are virtually the same as the relations (3.9).)

Proof of Theorem 4. For the proof we follow the excellent exposition of Berndt $[1, \mathrm{pp} .9-12]$. As noted earlier, the assumptions of the theorem imply directly that $\Phi(s)$ has at most finitely many simple poles at rational integral values of $s$. By the integral formula

$$
e^{-x}=\frac{1}{2 \pi i} \int_{d-i \infty}^{d+i \infty} \Gamma(s) x^{-s} d s, \quad(x, d>0)
$$

and absolute convergence of the Dirichlet series $\sum_{n=1}^{\infty} a_{n} n^{-s}$ for $\sigma=\operatorname{Re} s$ sufficiently large, it follows that for large positive $d, F(z)=F_{\Phi}(z)$ has a representation as the inverse Mellin transform of $\Phi(s)$ :

$$
F(i y)-a_{0}=\frac{1}{2 \pi i} \int_{d-i \infty}^{d+i \infty} \Phi(s) y^{-s} d s .
$$

For convenience we consider the cases $k \geq 0, k<0$ separately. Assume first that $k \geq 0$; it follows that (3.12) holds as well with $d$ replaced by $d+2 k$. We may suppose that $d$ is so large that all of the poles of $\Phi(s)$ lie between $-d$ and $d$. Following Hecke, we move 
the line of integration in (3.12) to $\operatorname{Re} s=-d$. This is accomplished by integrating on the boundary of a rectangle with vertices $\pm d \pm i T, T>0$, applying the residue theorem and assuming (as we shall show later) that the integrals on the horizontal sides tend to 0 as $T \rightarrow \infty$. We obtain

$$
F(i y)-a_{0}=\frac{1}{2 \pi i} \int_{-d-i \infty}^{-d+i \infty} \Phi(s) y^{-s} d s+\sum_{n=-[d]}^{[d]} \alpha_{n} y^{-n},
$$

where $\alpha_{n}$ is the residue of $\Phi(s)$ at $s=n$.

Apply the functional equation (3.3):

$$
\begin{aligned}
\frac{1}{2 \pi i} \int_{-d-i \infty}^{-d+i \infty} \Phi(s) y^{-s} d s=\frac{(-1)^{k}}{2 \pi i} \int_{-d-i \infty}^{-d+i \infty} \Phi(2 k-s) y^{-s} d s & =\frac{(-1)^{k}}{2 \pi i} \int_{(d+2 k)-i \infty}^{(d+2 k)+i \infty} \Phi(s) y^{s-2 k} d s \\
& =(-1)^{k} y^{-2 k}\left\{F(i / y)-a_{0}\right\},
\end{aligned}
$$

since (3.12) holds with $d$ replaced by $d+2 k$. From (3.13) we have

$$
F(i y)-a_{0}=(-1)^{k} y^{-2 k} F(i / y)-a_{0}(-1)^{k} y^{-2 k}+\sum_{n=-[d]}^{[d]} \alpha_{n} y^{-n} ;
$$

that is,

$$
z^{-2 k} F(-1 / z)=F(z)+a_{0}\left(z^{-2 k}-1\right)-\sum_{n} \alpha_{n}(i)^{n} z^{-n}
$$

holds for $z=i y, y>0$. By analytic continuation (3.14) holds for all $z$ in $\mathscr{H}$. Since the power series representation

$$
F(z)=a_{0}+\sum_{n=1}^{\infty} a_{n} e^{2 \pi i n z}
$$

shows that $F(z)$ is holomorphic in $\mathscr{H}$, it follows that $F(z)$ is an entire modular integral of weight $2 k$ with rational period function having poles only at 0 and $\infty$.

When $k<0$ the procedure is precisely the same, except we choose $d>0$ at the outset so large that, once again, all of the poles of $\Phi(s)$ lie between $-d$ and $d$ and, in addition, the representation (3.12) holds with $d$ replaced by $d+2 k$. It follows that (3.12) itself holds (since $d>d+2 k$ ) and we may proceed as before to derive (3.14).

It remains only to verify that the integrals

$$
\int_{d+i \mathrm{~T}}^{-d+i \mathrm{~T}} \Phi(s) y^{-s} d s \text { and } \int_{-d-i \mathrm{~T}}^{d-i \mathrm{~T}} \Phi(s) y^{-s} d s
$$

have limit 0 as $T \rightarrow \infty$. By assumption, $\Phi(s)$ is bounded in the truncated strip

$$
|\operatorname{Re} s| \leq d, \quad|\operatorname{Im} s| \geq t_{0}>0,
$$

but this by itself does not quite suffice. However, on the lines $\operatorname{Re} s=d$ and $\operatorname{Re} s=d+2 k$ the Dirichlet series $\phi(s)=\sum_{n=1}^{\infty} a_{n} n^{-s}$ is bounded, by the absolute convergence (uniform in 
$\operatorname{Im} s)$. Furthermore, since $\phi(s)=(2 \pi)^{s} \Gamma(s)^{-1} \Phi(s)$, Stirling's formula

$$
|\Gamma(\sigma+i t)| \sim(2 \pi)^{1 / 2}|t|^{\sigma-1 / 2} e^{-\pi|t| / 2}, \quad|t| \rightarrow \infty,
$$

which holds uniformly in a fixed interval $\sigma_{1} \leq \sigma \leq \sigma_{2}$, and the boundedness of $\Phi(s)$ imply that $\phi(s)=O\left(|t|^{1 / 2-\sigma} e^{-\pi \mid t / 22}\right),|t| \rightarrow \infty$, uniformly in the truncated strip (3.15). Since

$$
\phi(s)=(-1)^{k}(2 \pi)^{2 s-2 k} \Gamma(2 k-s) \phi(2 k-s) / \Gamma(s),
$$

we apply Stirling's formula to obtain $\phi(s)=O\left(1|t|^{2 k+2 d}\right),|t| \rightarrow \infty$, on the line $\operatorname{Re} s=-d$. The Phragmén-Lindelöf principle for the truncated vertical strip [10, p. 180] now implies that $\phi(s)=O\left(|t|^{K}\right),|t| \rightarrow \infty$, for some $K>0$, uniformly in the two half-strips defined by (3.15).

A final application of Stirling's formula implies that

$$
\Phi(s)=O\left(|T|^{d-1 / 2+K} e^{-\pi \mid T / 2}\right), \quad|T| \rightarrow \infty,
$$

uniformly on the intervals $[-d+i T, d+i T]$ and $[-d-i T, d-i T]$. Hence the integrals along these two paths approach 0 as $|T| \rightarrow \infty$. The proof of Theorem 4 is complete.

\section{REFERENCES}

1. B. Berndt, Hecke's theory of modular forms and Dirichlet series, Lecture notes, University of Illinois, Urbana (1970).

2. G. Bol, Invarianten linearer Differentialgleichungen, Abh. Math. Sem. Univ. Hamburg 16 (1949), nos. 3-4, 1-28.

3. H. Cohn and M. I. Knopp, Note on automorphic forms with real period polynomials, Duke Math. J. 32 (1965), 115-120. 298.

4. M. Eichler, Eine Verallgemeinerung der Abelschen Integrale, Math. Z. 67 (1957), 267-

5. A. Hurwitz, Grundlagen einer independenten Theorie der elliptischen Modulfunctionen und Theorie der Multiplicatorgleichungen erster Stufe, Math. Annalen 18 (1881), 528-591.

6. S. Y. Husseini and M. I. Knopp, Eichler cohomology and automorphic forms, Illinois $J$. Math. 15 (1971), 565-577. $47-62$.

7. M. I. Knopp, Rational period functions of the modular group, Duke Math. J. 45 (1978),

8. - Some new results on the Eichler cohomology of automorphic forms, Bull. Amer. Math. Soc. 80 (1974), 607-632.

9. L. A. Parson and K. Rosen, Automorphic integrals and rational period functions for the Hecke groups, to appear.

10. E. C. Titchmarsh, The Theory of Functions, 2nd edition (Oxford University Press, 1939).

TEMPLE UNIVERSTTY

PhiladelPhia 